\title{
OPEN The age distribution of facial metrics in two large Korean populations
}

Received: 29 January 2019

Accepted: 25 September 2019

Published online: 10 October 2019

\author{
Hae-Young Lee ${ }^{1}$, Seongwon Cha², Hyo-Jeong Ban², In-Young Kim¹, Bo-Reum Park ${ }^{1}$, \\ Ig-Jae Kim ${ }^{3} \&$ Kyung-Won Hong ${ }^{1}$
}

Growth and alterations in craniofacial morphology have attracted interest in many fields of science, especially physical anthropology, genetics and forensic sciences. We performed an analysis of craniofacial morphology alterations by gender and ageing stage in Korean populations. We studied 15 facial metrics using two large Korean populations (1,926 samples from the Korea Medicine Data Center cohort and 5,643 samples from the Ansan-Ansung cohort). Among the 15 metrics, 12 showed gender differences and tended to change with age. In both of the independent populations, brow ridge height, upper lip height, nasal tip height, and profile nasal length tended to increase with age, whereas outer canthal width, right palpebral fissure height, left palpebral fissure height, right upper lip thickness, left upper lip thickness, nasal tip protrusion, facial base width, and lower facial width tended to decrease. In conclusion, our findings suggest that ageing (past 40 years of age) might affect eye size, nose length, upper lip thickness, and facial width, possibly due to loss of elasticity in the face. Therefore, these facial metric changes could be applied to individual age prediction and aesthetic facial care.

The appearance of the face typically changes in different dimensions and directions with age ${ }^{1,2}$. This phenomenon, known as allometry, is the reason why young faces are distinct from old faces ${ }^{3}$. Growth and alteration in craniofacial morphology have generated interest in many fields of science, especially physical anthropology ${ }^{4,5}$ and genet$\mathrm{ics}^{6,7}$. Additionally, alternative applications of craniofacial morphology, such as forensic science, have received considerable attention ${ }^{8}$. In the field of Korean Oriental medicine, facial metrics are regarded as representative and reliable characteristics for diagnosing a person's Sasang constitution ${ }^{9}$. Previously, we performed a two-stage genome-wide association study of facial morphological traits in two large Korean populations (1,926 samples from the Korea Medicine Data Center (KDC) cohort and 5,643 samples from the Ansan-Ansung cohort) ${ }^{6}$.

We speculated that the ageing-related trends in each facial metric might be important for human evolution and appearance. In this study, we compared 15 facial metrics - (a) BRH, brow ridge height; (b) IW, intercanthal width; (c) OW, outer canthal width; (d) RPFH, right palpebral fissure height; (e) LPFH, left palpebral fissure height; (f) NBH, nasal bridge height; (g) ULH, upper lip height; (h) RULT, right upper lip thickness; (i) LULT, left upper lip thickness; (j) SW, subnasal width; (k) PNL, profile nasal length; (l) FBW, facial base width; (m) NTH(V), nasal tip height (vertical); (n) NTP(H), nasal tip protrusion (horizontal); and (o) LFW, lower facial width - for each of four age groups ( $40 \mathrm{~s}, 50 \mathrm{~s}, 60 \mathrm{~s}$ and over $70 \mathrm{~s}$ ) using the KDC and Ansan-Ansung cohorts as the replication set (Fig. 1). For example, the brow ridge is the nodule or crest of bone that is situated on the frontal bone of the skull $^{10}$, forming the boundary between the forehead itself and the tops of the eye sockets. Normally, in humans, the ridges arch over each eye, offering mechanical protection ${ }^{11}$. Typically, the arches are more prominent in men than in women and vary between different ethnic groups ${ }^{12}$.

\footnotetext{
${ }^{1}$ Theragenetex Bioinstitute Co. Ltd, Suwon, 16229, Republic of Korea. ${ }^{2}$ Future Medicine Division, Korea Institute of Oriental Medicine, Daejeon, 34054, Republic of Korea. ${ }^{3}$ Center for Imaging Media Research, Korea Institute of Science and Technology, Seoul, 02792, Republic of Korea. Hae-Young Lee and Seongwon Cha contributed equally. Correspondence and requests for materials should be addressed to K.-W.H. (email: kyungwon.hong@theragenetex. com)
} 

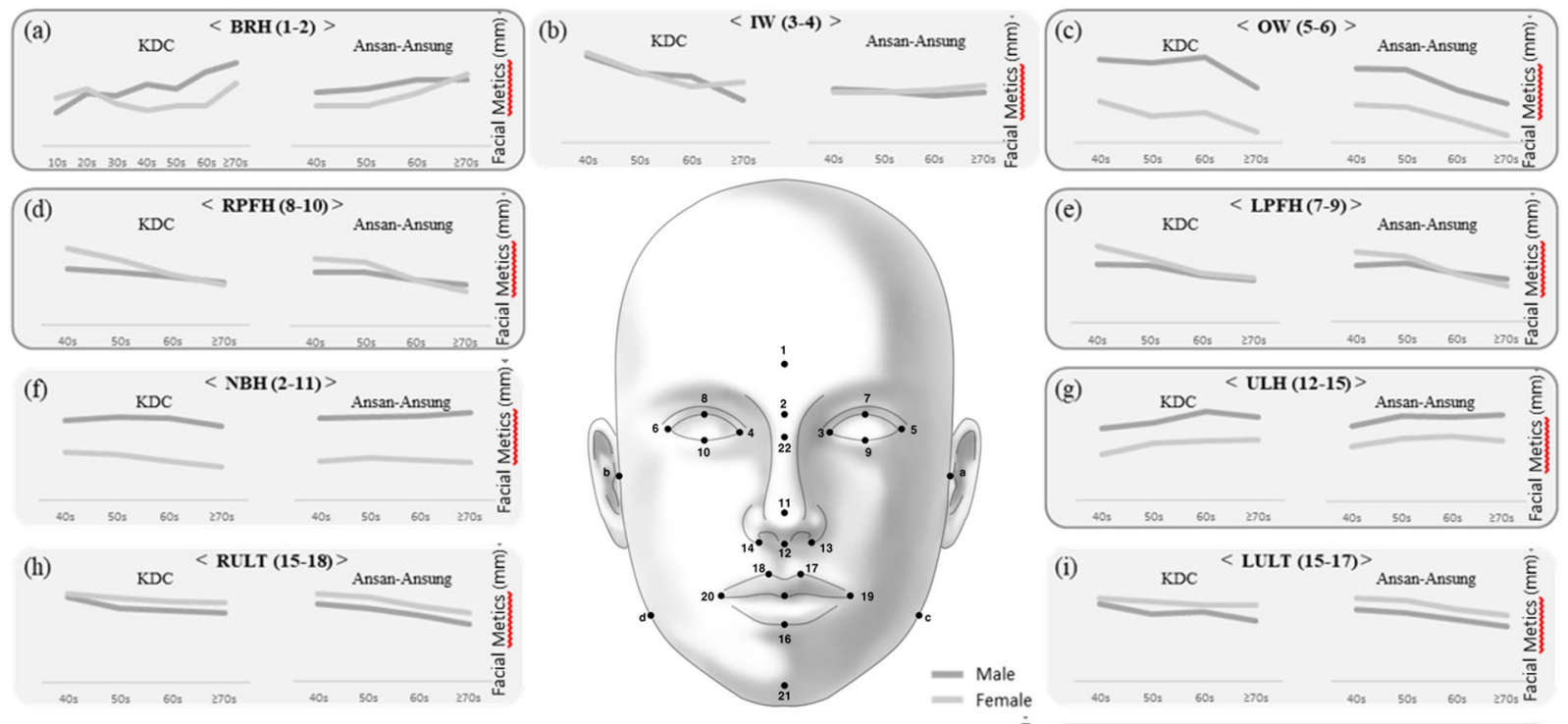

(i)

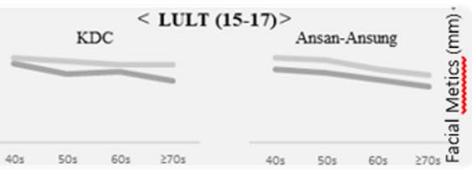

(j)
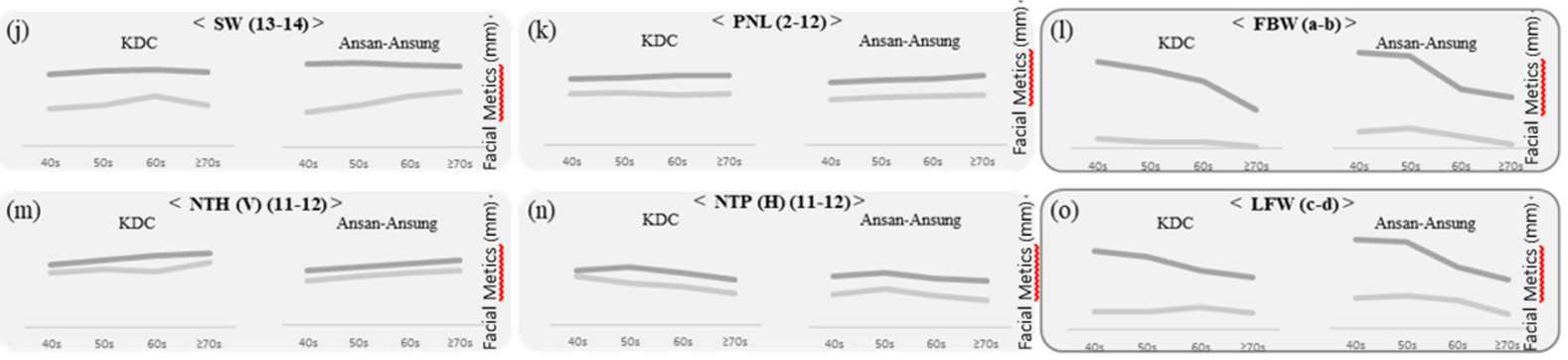

Figure 1. Facial changes from age 40 years to age $70+$ years. (a) BRH, brow ridge height; (b) IW, intercanthal width; (c) OW, outer canthal width; (d) RPFH, right palpebral fissure height; (e) LPFH, left palpebral fissure height; (f) NBH, nasal bridge height; (g) ULH, upper lip height; (h) RULT, right upper lip thickness; (i) LULT, left upper lip thickness; (j) SW, subnasal width; (k) PNL, profile nasal length; (l) FBW, facial base width; (m) $\mathrm{NTH}(\mathrm{V})$, nasal tip height; (n) NTP(H), nasal tip protrusion; and (o) LFW, lower facial width.

\section{Results}

Gender differences. The distribution characteristics of 15 facial metrics are described in Table 1 and plotted in Fig. 1 by gender and age group in the KDC and Ansan-Ansung cohorts. The prominent gender differences were identified by the sex differences in the overall sample, as shown in Table 1, and the age distribution of sex differences, as shown in Fig. 1. Among the 15 facial metrics, six metrics (OW, NBH, ULH, SW, FBW, and LFW) appeared to be greater in males than in females in the overall sample comparison in both the KDC and AnsanAnsung cohorts. In the age distribution of gender differences in Fig. 1, 12 metrics (BRH, OW, NBH, ULH, RPFH, LPFH, SW, PNL, FBW, NTH(V), NTP (H), LFW) showed gender differences. Interestingly, 4 metrics (RPFH, LPFH, RULT, LULT) were larger in females than in males.

Age group comparison. Age group differences were analysed by Student's t-tests comparing the $40 \mathrm{~s}$ age group to the $50 \mathrm{~s}, 60 \mathrm{~s}$ and $70 \mathrm{~s}$ age groups. BRH, ULH, and NTH (v) are slightly increased in the $60 \mathrm{~s}$ age group in both cohorts and in both genders (Fig. 1a,g,m). OW, RULT, LULT, and NTP (H) were slightly decreased in the $60 \mathrm{~s}$ age group in both cohorts and in both genders (Fig. 1c,h,I,n). RPFH and LPFH showed a considerably decreased pattern in both cohorts and in both genders, and the pattern was prominent in females in both cohorts (Fig. 1d,e). Although FBW and LFW showed a considerably decreased pattern in both cohorts and in both genders, the pattern was prominent in males in both cohorts (Fig. 1l,o). PNL showed a slight increase in both cohorts, but only in males (Fig. 1k). IW, NBH, and SW seemed to have no clear pattern (Fig. 1b,f,j).

To understand in greater detail the effect of ageing on facial metrics, we constructed linear regression models with age and/or body mass index as covariates. We identified a significant linear increasing tendency of BRH and NTP and a decreasing tendency of OW, RPFH, LPFH, RULT, LULT, NTH, and NTP in both cohorts and in both sexes (Table 1).

Ethnic comparison of the human orbital region. Facial metrics in the orbital region, including PFH, IW, and OW, were compared between Koreans and other ethnicities (Fig. 2). RPFH and LPFH were relatively low among non-Asians relative to Asians (Fig. 2A). The Korean subjects had a large OW, ranking between those of Japanese and Indian individuals, whereas the IW was of Koreans was smaller than those of other Asian populations, such as Japanese and Chinese (Fig. 2B,C). Interestingly, the gender difference in IW was no more prominent in Koreans than in other ethnic groups. 


\begin{tabular}{|c|c|c|c|c|c|c|c|c|c|c|c|c|c|c|c|c|c|c|c|c|c|c|c|c|c|c|}
\hline \multirow[b]{3}{*}{$\begin{array}{l}\text { Coordi- } \\
\text { nate* }\end{array}$} & \multirow[b]{3}{*}{$\begin{array}{l}\text { Pheno- } \\
\text { type }\end{array}$} & \multirow[b]{3}{*}{$\begin{array}{l}\text { Phenotype } \\
\text { description }\end{array}$} & \multirow[b]{3}{*}{ Sex } & \multirow[b]{3}{*}{$\begin{array}{l}\text { Popu- } \\
\text { lation }\end{array}$} & \multicolumn{15}{|c|}{ Means and standard deviations of facial metrics in each age group (mm) } & \multirow{3}{*}{\begin{tabular}{|l|}
$\begin{array}{l}\text { ANOVA } \\
\text { test }\end{array}$ \\
p-value \\
\end{tabular}} & \multicolumn{6}{|c|}{ Linear regression } \\
\hline & & & & & \multicolumn{4}{|l|}{ Total } & \multicolumn{3}{|l|}{40} & \multicolumn{3}{|l|}{50} & \multicolumn{3}{|l|}{60} & 70 & & & Adjuste & ted for $a$ & & $\begin{array}{l}\text { Adjuste } \\
\text { BMI }\end{array}$ & ed by ag & ge and \\
\hline & & & & & $\mathbf{N}$ & Mean & SD & $\mathbf{N}$ & Mean & SD & $\mathbf{N}$ & Mean & SD & $\mathbf{N}$ & Mean & SD & $\mathbf{N}$ & Mean & SD & & Beta & \begin{tabular}{|l|}
$\begin{array}{l}\text { Std. } \\
\text { error }\end{array}$ \\
\end{tabular} & Sig. & Beta & \begin{tabular}{|l|} 
Std. \\
error
\end{tabular} & Sig. \\
\hline & & & & KDC & 670 & 29.8 & 4.3 & 145 & 29.8 & 4 & 147 & 29.5 & \begin{tabular}{|l|}
4.6 \\
\end{tabular} & 106 & 30.6 & 4.5 & 73 & 31.1 & 4.9 & $<0.05$ & 0.10 & 0.02 & $<0.05$ & 0.11 & \begin{tabular}{|l|}
0.02 \\
\end{tabular} & $<0.05$ \\
\hline (a) & BPH & Brov & Male & $\begin{array}{l}\text { Ansan- } \\
\text { Ansung }\end{array}$ & 2541 & 29.7 & 4.3 & 245 & 29.3 & 3.8 & 1222 & 29.5 & 4 & 658 & 30.1 & 4.8 & 416 & 30 & 4.7 & $<0.01$ & 0.07 & 0.01 & $<0.001$ & 0.11 & 0.01 & $<0.001$ \\
\hline (a) & BRH & & & KDC & 1164 & 28.7 & 4 & 255 & 28.2 & 3.6 & 250 & \begin{tabular}{|l|}
28.5 \\
\end{tabular} & \begin{tabular}{|l|}
3.9 \\
\end{tabular} & 178 & $\mid 28.5$ & 4.4 & \begin{tabular}{|l|}
123 \\
\end{tabular} & 29.9 & 5 & $<0.01$ & 0.12 & 0.01 & $<0.01$ & 0.10 & 0.01 & $<0.01$ \\
\hline & & & Female & $\begin{array}{l}\text { Ansan- } \\
\text { Ansung }\end{array}$ & 2721 & 29 & 3.9 & 216 & 28.4 & 3.6 & 1191 & 28.4 & 3.5 & 808 & 29.2 & 3.9 & 506 & 30.4 & 4.3 & $<0.001$ & 0.18 & 0.01 & $<0.001$ & 0.18 & 0.01 & $<0.001$ \\
\hline & & & & KDC & 708 & 36.5 & 3.8 & 155 & 36.6 & 3.6 & 160 & 35.9 & \begin{tabular}{|l|}
3.3 \\
\end{tabular} & 113 & \begin{tabular}{|l|}
35.7 \\
\end{tabular} & 4.1 & 74 & 34.8 & 4.5 & $<0.01$ & -0.18 & 0.02 & $<0.001$ & -0.17 & \begin{tabular}{|l|}
0.02 \\
\end{tabular} & $<0.001$ \\
\hline (b) & IW & Inter & Male & $\begin{array}{l}\text { Ansan- } \\
\text { Ansung }\end{array}$ & 2604 & 35.6 & 3.8 & 248 & 35.9 & 3.3 & 1252 & 35.7 & 3.5 & 674 & 35.4 & 3.9 & 430 & 35.7 & 4.5 & 0.233 & -0.03 & 0.01 & 0.16 & -0.03 & 0.01 & 0.13 \\
\hline (b) & IW & w & & KDC & 1263 & 36.4 & 3.5 & 275 & 36.7 & 3.3 & 274 & 35.9 & 3.6 & 190 & \begin{tabular}{|l|}
35.3 \\
\end{tabular} & 3.8 & \begin{tabular}{|l|}
131 \\
\end{tabular} & 35.5 & 3.5 & $<0.001$ & -0.15 & 0.01 & $<0.001$ & -0.15 & 0.01 & $<0.001$ \\
\hline & & & Female & \begin{tabular}{|l|} 
Ansan- \\
Ansung
\end{tabular} & 2978 & 35.8 & 3.5 & 245 & 35.6 & 3 & 1286 & 35.6 & 3.1 & 871 & 35.8 & 3.6 & 576 & 36.2 & 4.2 & $<0.01$ & 0.05 & 0.01 & $<0.05$ & 0.05 & 0.01 & $<0.01$ \\
\hline & & & & KDC & 716 & \begin{tabular}{|l|}
99.7 \\
\end{tabular} & 7 & 156 & 99.7 & 6.7 & 160 & \begin{tabular}{|l|}
99.4 \\
\end{tabular} & \begin{tabular}{|l|}
6.8 \\
\end{tabular} & 117 & \begin{tabular}{|l|}
99.9 \\
\end{tabular} & 7.2 & \begin{tabular}{|l|}
77 \\
\end{tabular} & 97 & 8.7 & $<0.05$ & -0.12 & 0.03 & $<0.01$ & -0.10 & \begin{tabular}{|l|}
0.03 \\
\end{tabular} & $<0.05$ \\
\hline (c) & ow & Outer & Male & $\begin{array}{l}\text { Ansan- } \\
\text { Ansung }\end{array}$ & 2624 & 99 & 6.5 & 249 & 100.4 & 5.2 & 1254 & 100.3 & 5.9 & 686 & 97.9 & 6.6 & 435 & 96.4 & 7.3 & $<0.001$ & -0.25 & 0.01 & $<0.001$ & -0.17 & 0.01 & $<0.001$ \\
\hline (c) & $0 \mathrm{w}$ & $\begin{array}{l}\text { cantnat } \\
\text { width }\end{array}$ & & KDC & \begin{tabular}{|l|}
1275 \\
\end{tabular} & \begin{tabular}{|l|}
95.3 \\
\end{tabular} & 6.2 & 275 & \begin{tabular}{|l|}
95.8 \\
\end{tabular} & 5.2 & 274 & \begin{tabular}{|l|}
94.5 \\
\end{tabular} & 6.1 & 193 & \begin{tabular}{|l|}
94.8 \\
\end{tabular} & 7 & \begin{tabular}{|l|l}
140 \\
\end{tabular} & 93 & 7.3 & $<0.001$ & -0.14 & 0.02 & $<0.001$ & -0.16 & 0.02 & $<0.001$ \\
\hline & & & Female & $\begin{array}{l}\text { Ansan- } \\
\text { Ansung }\end{array}$ & 2982 & 95 & 5.9 & 246 & 96.3 & 4.9 & 1286 & 96.1 & 5.3 & 874 & 94.6 & 6 & 576 & 92.9 & 6.8 & $<0.001$ & -0.21 & 0.01 & $<0.001$ & -0.22 & 0.01 & $<0.001$ \\
\hline & & & & KDC & 717 & 7.7 & 1.4 & 155 & 7.9 & 1.4 & 162 & 7.7 & 1.5 & 117 & 7.5 & 1.3 & \begin{tabular}{|l|l}
77 \\
\end{tabular} & 7.2 & 1.5 & $<0.001$ & -0.17 & 0.01 & $<0.001$ & -0.18 & 0.01 & $<0.001$ \\
\hline (d) & RPFH & $\begin{array}{l}\text { Right } \\
\text { palpebral }\end{array}$ & Male & $\begin{array}{l}\text { Ansan- } \\
\text { Ansung }\end{array}$ & 2629 & 7.5 & 1.4 & 249 & 7.8 & 1.4 & 1254 & 7.7 & 1.4 & 689 & 7.3 & 1.5 & 437 & 7.1 & 1.5 & $<0.001$ & -0.19 & 0.00 & $<0.001$ & -0.21 & 0.00 & $<0.001$ \\
\hline (d) & RPFH & fissure & & KDC & 1277 & 8.5 & 1.7 & 275 & 8.9 & 1.5 & 276 & 8.3 & 1.6 & 195 & 7.6 & 1.5 & 141 & 7 & 1.7 & $<0.001$ & -0.40 & 0.00 & $<0.001$ & -0.39 & 0.00 & $<0.001$ \\
\hline & & & Female & $\begin{array}{l}\text { Ansan- } \\
\text { Ansung }\end{array}$ & 2986 & 7.7 & 1.6 & 245 & 8.5 & 1.4 & 1285 & 8.3 & 1.4 & 876 & 7.4 & 1.5 & 580 & 6.8 & 1.4 & $<0.001$ & -0.40 & 0.00 & $<0.001$ & -0.40 & 0.00 & $<0.001$ \\
\hline & & & & KDC & 715 & 7.9 & 1.5 & 155 & 8 & 1.4 & 162 & 8 & 1.6 & 116 & \begin{tabular}{|l|}
7.4 \\
\end{tabular} & 1.4 & \begin{tabular}{|l|l}
75 \\
\end{tabular} & 7.2 & 1.6 & $<0.001$ & -0.21 & 0.01 & $<0.001$ & -0.22 & 0.01 & $<0.001$ \\
\hline (e) & IPFH & $\begin{array}{l}\text { Left } \\
\text { palpebral }\end{array}$ & Male & $\begin{array}{l}\text { Ansan- } \\
\text { Ansung }\end{array}$ & 2626 & 7.7 & 1.5 & 250 & 7.9 & 1.3 & 1252 & 8 & 1.4 & 688 & 7.5 & 1.5 & 436 & 7.2 & 1.6 & $<0.001$ & -0.21 & 0.00 & $<0.001$ & -0.22 & 0.00 & $<0.001$ \\
\hline (e) & LPFH & fissure & & KDC & 1279 & 8.5 & 1.7 & 275 & 8.9 & 1.4 & 276 & 8.3 & 1.6 & 194 & 7.6 & 1.7 & 142 & 7.3 & 1.6 & $<0.001$ & -0.37 & 0.00 & $<0.001$ & -0.35 & 0.00 & $<0.001$ \\
\hline & & & Female & $\begin{array}{l}\text { Ansan- } \\
\text { Ansung }\end{array}$ & 2977 & 7.8 & 1.6 & 246 & 8.6 & 1.5 & 1283 & 8.4 & 1.5 & 872 & 7.4 & 1.5 & 576 & 6.9 & 1.6 & $<0.001$ & -0.40 & 0.00 & $<0.001$ & -0.40 & 0.00 & $<0.001$ \\
\hline & & & & KDC & 680 & 35.8 & 4.1 & 150 & 35.8 & 3.9 & 149 & 36 & \begin{tabular}{|l|}
3.7 \\
\end{tabular} & 110 & 35.9 & 4.5 & 74 & 35.4 & 4.4 & 0.609 & -0.02 & 0.02 & 0.59 & -0.03 & \begin{tabular}{|l|}
0.02 \\
\end{tabular} & 0.48 \\
\hline (f) & $\mathrm{NBH}$ & Nasa & Male & $\begin{array}{l}\text { Ansan- } \\
\text { Ansung }\end{array}$ & 2561 & 36.1 & 3.9 & 246 & 35.9 & 3.5 & 1229 & 36 & 3.8 & 667 & 36.1 & 4 & 419 & 36.4 & 4.4 & 0.112 & 0.04 & 0.01 & $<0.05$ & 0.00 & 0.01 & 0.89 \\
\hline (I) & NDH & & & KDC & 1210 & 33.3 & 3.8 & 264 & 33.5 & 3.4 & 260 & 33.3 & 3.9 & 185 & 32.8 & 3.8 & 134 & 32.4 & 4.3 & $<0.01$ & -0.10 & 0.01 & $<0.01$ & -0.09 & 0.01 & $<0.05$ \\
\hline & & & Female & $\begin{array}{l}\text { Ansan- } \\
\text { Ansung }\end{array}$ & 2826 & 32.9 & 3.6 & 230 & 32.8 & 3.5 & 1224 & 33 & 3.4 & 831 & 32.9 & 3.7 & 541 & 32.8 & 4 & 0.372 & -0.02 & 0.01 & 0.41 & -0.01 & 0.01 & 0.52 \\
\hline & & & & KDC & 675 & 29.5 & 3 & 147 & 29.4 & 2.9 & 154 & 29.8 & 2.9 & 112 & 30.5 & 3.2 & 67 & 30.1 & 3.6 & $<0.05$ & 0.11 & 0.01 & $<0.05$ & 0.12 & 0.01 & $<0.01$ \\
\hline (a) & UUH & Upp & Male & \begin{tabular}{|l|} 
Ansan- \\
Ansung
\end{tabular} & 2524 & 30.2 & 2.8 & 242 & 29.6 & 2.5 & 1226 & 30.3 & 2.7 & 649 & 30.2 & 2.9 & 407 & 30.4 & 3.2 & 0.0612 & 0.04 & 0.01 & 0.05 & 0.05 & 0.01 & $<0.01$ \\
\hline (g) & OLH & & & KDC & 1252 & 27.9 & 2.6 & 270 & 27.8 & 2.3 & 271 & 28.5 & 2.6 & 190 & 28.6 & 2.7 & \begin{tabular}{|l|}
138 \\
\end{tabular} & 28.7 & 3.1 & $<0.001$ & 0.11 & 0.01 & $<0.001$ & 0.10 & 0.01 & $<0.01$ \\
\hline & & & Female & $\begin{array}{l}\text { Ansan- } \\
\text { Ansung }\end{array}$ & 2941 & 28.9 & 2.6 & 243 & 28.4 & 2.2 & 1279 & 28.9 & 2.3 & 859 & 29.1 & 2.7 & 560 & 28.8 & 3 & 0.245 & 0.02 & 0.01 & 0.29 & 0.02 & 0.01 & 0.33 \\
\hline & & & & KDC & 677 & 2.1 & 0.2 & 150 & 2.2 & 0.2 & 155 & 2.1 & 0.2 & 108 & 2.1 & 0.3 & 66 & 2 & 0.3 & $<0.001$ & -0.18 & 0.00 & $<0.001$ & -0.18 & 0.00 & $<0.001$ \\
\hline (b) & RUUT & Right & Male & $\begin{array}{l}\text { Ansan- } \\
\text { Ansung }\end{array}$ & 2476 & 2.1 & 0.2 & 242 & 2.1 & 0.2 & 1216 & 2.1 & 0.2 & 631 & 2 & 0.2 & 387 & 2 & 0.3 & $<0.001$ & -0.22 & 0.00 & $<0.001$ & -0.22 & 0.00 & $<0.001$ \\
\hline (h) & RULT & $\begin{array}{l}\text { upper lip } \\
\text { thickness }\end{array}$ & & KDC & 1256 & 2.2 & 0.2 & 272 & 2.2 & 0.2 & 272 & 2.1 & 0.2 & 190 & 2.1 & 0.2 & 133 & 2.1 & 0.2 & $<0.001$ & -0.13 & 0.00 & $<0.001$ & -0.13 & 0.00 & $<0.001$ \\
\hline & & & Female & $\begin{array}{l}\text { Ansan- } \\
\text { Ansung }\end{array}$ & 2941 & 2.1 & 0.2 & 245 & 2.2 & 0.2 & 1282 & 2.2 & 0.2 & 855 & 2.1 & 0.2 & 559 & 2.1 & 0.3 & $<0.001$ & -0.25 & 0.00 & $<0.001$ & -0.25 & 0.00 & $<0.001$ \\
\hline & & & & KDC & 683 & 2.1 & 0.2 & 152 & 2.1 & 0.2 & 153 & 2.1 & 0.2 & 108 & 2.1 & 0.2 & 69 & 2 & 0.3 & $<0.001$ & -0.18 & 0.00 & $<0.001$ & -0.18 & 0.00 & $<0.001$ \\
\hline (i) & IUUT & Left & Male & $\begin{array}{l}\text { Ansan- } \\
\text { Ansung }\end{array}$ & 2476 & 2 & 0.2 & 241 & 2.1 & 0.2 & 1212 & 2.1 & 0.2 & 634 & 2 & 0.2 & 389 & 2 & 0.3 & $<0.001$ & -0.19 & 0.00 & $<0.001$ & -0.19 & 0.00 & $<0.001$ \\
\hline (i) & & lip $t$ & & KDC & 1255 & 2.2 & 0.2 & 272 & 2.2 & 0.2 & 272 & 2.2 & 0.2 & 190 & 2.1 & 0.2 & 132 & 2.1 & 0.2 & $<0.01$ & -0.12 & 0.00 & $<0.001$ & -0.13 & 0.00 & $<0.001$ \\
\hline & & & Female & $\begin{array}{l}\text { Ansan- } \\
\text { Ansung }\end{array}$ & 2946 & 2.1 & 0.2 & 244 & 2.2 & 0.2 & 1283 & 2.2 & 0.2 & 859 & 2.1 & 0.2 & 560 & 2 & 0.3 & $<0.001$ & -0.25 & 0.00 & $<0.001$ & -0.25 & 0.00 & $<0.001$ \\
\hline & & & & KDC & 682 & 27 & 3 & 148 & 27 & 2.8 & 157 & 27.3 & 3.3 & 109 & 27.4 & 2.9 & \begin{tabular}{|l|l}
72 \\
\end{tabular} & 27.3 & 2.5 & 0.437 & 0.02 & 0.01 & 0.60 & 0.03 & \begin{tabular}{|l|}
0.01 \\
\end{tabular} & 0.46 \\
\hline (i) & sw & Subnasal & Male & $\begin{array}{l}\text { Ansan- } \\
\text { Ansung }\end{array}$ & 2556 & 27.9 & 2.6 & 244 & 27.9 & 2.7 & 1228 & 28 & 2.7 & 661 & 27.9 & 2.6 & 423 & 27.7 & 2.5 & 0.0811 & -0.04 & 0.01 & $<0.05$ & -0.01 & 0.01 & 0.51 \\
\hline (j) & & & & KDC & 1253 & 24.6 & 2.7 & 269 & 24.6 & 2.5 & 272 & 24.8 & 2.6 & 192 & 25.5 & 2.6 & 136 & 24.9 & 2.8 & $<0.05$ & 0.09 & 0.01 & $<0.05$ & 0.06 & \begin{tabular}{|l|}
0.01 \\
\end{tabular} & 0.07 \\
\hline & & & Female & $\begin{array}{l}\text { Ansan- } \\
\text { Ansung }\end{array}$ & 2957 & 25.3 & 2.4 & 244 & 24.5 & 2.2 & 1280 & 25 & 2.2 & 867 & 25.6 & 2.4 & 566 & 26 & 2.5 & $<0.001$ & 0.20 & 0.01 & $<0.001$ & 0.19 & 0.00 & $<0.001$ \\
\hline & & & & KDC & 685 & 3.9 & 0.1 & 151 & 3.9 & 0.1 & 149 & 3.9 & \begin{tabular}{|l|}
0.1 \\
\end{tabular} & 111 & 4 & 0.1 & 75 & 4 & 0.1 & $<0.05$ & 0.08 & 0.00 & 0.08 & 0.08 & \begin{tabular}{|l|}
0.00 \\
\end{tabular} & 0.09 \\
\hline ok & PNL & Profile & Male & $\begin{array}{l}\text { Ansan- } \\
\text { Ansung }\end{array}$ & 2566 & 3.9 & 0.1 & 244 & 3.9 & 0.1 & 1235 & 3.9 & 0.1 & 669 & 3.9 & 0.1 & 418 & 4 & 0.1 & $<0.001$ & 0.13 & 0.00 & $<0.001$ & 0.12 & 0.00 & $<0.001$ \\
\hline (k) & & & & KDC & \begin{tabular}{|l|}
1211 \\
\end{tabular} & 3.9 & 0.1 & 263 & 3.9 & \begin{tabular}{l|l}
0.1 \\
\end{tabular} & 262 & 3.9 & \begin{tabular}{|l|}
0.1 \\
\end{tabular} & 182 & 3.9 & 0.1 & \begin{tabular}{|l|}
135 \\
\end{tabular} & 3.9 & 0.1 & 0.556 & -0.02 & 0.00 & 0.50 & -0.02 & \begin{tabular}{|l|}
0.00 \\
\end{tabular} & 0.56 \\
\hline & & & Female & \begin{tabular}{|l|} 
Ansan- \\
Ansung
\end{tabular} & 2809 & 3.8 & 0.1 & 226 & 3.8 & 0.1 & 1228 & 3.8 & 0.1 & 832 & 3.8 & 0.1 & 523 & 3.9 & 0.1 & $<0.001$ & 0.09 & 0.00 & $<0.001$ & 0.09 & 0.00 & $<0.001$ \\
\hline
\end{tabular}




\begin{tabular}{|c|c|c|c|c|c|c|c|c|c|c|c|c|c|c|c|c|c|c|c|c|c|c|c|c|c|c|}
\hline \multirow[b]{3}{*}{$\begin{array}{l}\text { Coordi- } \\
\text { nate* }\end{array}$} & \multirow[b]{3}{*}{$\begin{array}{l}\text { Pheno- } \\
\text { type }\end{array}$} & \multirow[b]{3}{*}{$\begin{array}{l}\text { Phenotype } \\
\text { description }\end{array}$} & \multirow[b]{3}{*}{ Sex } & \multirow[b]{3}{*}{$\begin{array}{l}\text { Popu- } \\
\text { lation }\end{array}$} & \multicolumn{15}{|c|}{ Means and standard deviations of facial metrics in each age group (mm) } & \multirow{3}{*}{\begin{tabular}{|l|}
$\begin{array}{l}\text { ANOVA } \\
\text { test }\end{array}$ \\
p-value \\
\end{tabular}} & \multicolumn{6}{|c|}{ Linear regression } \\
\hline & & & & & \multicolumn{4}{|l|}{ Total } & \multicolumn{3}{|l|}{40} & \multicolumn{3}{|l|}{50} & \multicolumn{3}{|l|}{60} & \multicolumn{2}{|l|}{70} & & \multicolumn{3}{|c|}{ Adjusted for age } & \multicolumn{3}{|c|}{$\begin{array}{l}\text { Adjusted by age and } \\
\text { BMI }\end{array}$} \\
\hline & & & & & $\mathbf{N}$ & Mean & SD & $\mathbf{N}$ & Mean & SD & $\mathbf{N}$ & Mean & SD & $\mathbf{N}$ & Mean & SD & $\mathbf{N}$ & Mean & SD & & Beta & \begin{tabular}{|l|}
$\begin{array}{l}\text { Std. } \\
\text { error }\end{array}$ \\
\end{tabular} & Sig. & Beta & \begin{tabular}{|l|} 
Std. \\
error
\end{tabular} & Sig. \\
\hline \multirow{4}{*}{ (1) } & \multirow{4}{*}{ FBW } & \multirow{4}{*}{$\begin{array}{l}\text { Facial base } \\
\text { width }\end{array}$} & \multirow[b]{2}{*}{ Male } & KDC & 703 & 156 & 9.1 & 154 & 157.6 & 9.1 & 159 & \begin{tabular}{|l|}
156.7 \\
\end{tabular} & \begin{tabular}{|l|}
9.3 \\
\end{tabular} & 117 & 155.2 & \begin{tabular}{|l|}
8.7 \\
\end{tabular} & 76 & \begin{tabular}{|l|} 
\\
\end{tabular} & 10.4 & $<0.001$ & -0.22 & \begin{tabular}{|l|}
0.04 \\
\end{tabular} & $<0.001$ & -0.20 & \begin{tabular}{|l|}
0.03 \\
\end{tabular} & $<0.001$ \\
\hline & & & & \begin{tabular}{|l|} 
Ansan- \\
Ansung
\end{tabular} & 2609 & 156.4 & 8.6 & 246 & 158.7 & 7.4 & 1244 & 158.2 & 8.1 & 681 & 154.3 & 8.5 & 438 & 153.3 & 9.4 & $<0.001$ & -0.26 & 0.02 & $<0.001$ & -0.13 & 0.02 & $<0.001$ \\
\hline & & & \multirow[b]{2}{*}{ Female } & KDC & 1258 & 148.1 & 7.6 & 271 & 148.3 & 6.9 & 269 & \begin{tabular}{|l|}
147.8 \\
\end{tabular} & 7.4 & 194 & 147.8 & 8.4 & 142 & 147.3 & 9.3 & 0.237 & -0.04 & 0.02 & 0.23 & -0.09 & \begin{tabular}{|l|}
0.02 \\
\end{tabular} & $<0.01$ \\
\hline & & & & \begin{tabular}{|l|} 
Ansan- \\
Ansung
\end{tabular} & 2975 & 148.8 & 7.4 & 245 & 149 & 6.8 & 1281 & 149.5 & 6.9 & 869 & 148.6 & 7.5 & 580 & 147.6 & 8.4 & $<0.001$ & -0.09 & 0.02 & $<0.001$ & -0.10 & 0.01 & $<0.001$ \\
\hline \multirow{4}{*}{$(\mathrm{m})$} & \multirow{4}{*}{$\begin{array}{l}\text { NTH } \\
\text { (V) }\end{array}$} & \multirow{4}{*}{$\begin{array}{l}\text { Nasal tip } \\
\text { height }\end{array}$} & \multirow[b]{2}{*}{ Male } & KDC & 689 & 2.7 & 0.2 & 148 & 2.6 & \begin{tabular}{|l|}
0.2 \\
\end{tabular} & 153 & 2.7 & \begin{tabular}{|l|}
0.1 \\
\end{tabular} & 116 & 2.7 & 0.2 & 76 & 2.7 & 0.2 & $<0.001$ & 0.18 & 0.00 & $<0.001$ & 0.19 & \begin{tabular}{|l|}
0.00 \\
\end{tabular} & $<0.001$ \\
\hline & & & & \begin{tabular}{|l|} 
Ansan- \\
Ansung \\
\end{tabular} & 2563 & 2.6 & 0.1 & 245 & 2.6 & 0.1 & 1236 & 2.6 & 0.1 & 666 & 2.6 & 0.1 & 416 & 2.7 & 0.2 & $<0.001$ & 0.15 & 0.00 & $<0.001$ & 0.18 & 0.00 & $<0.001$ \\
\hline & & & \multirow[b]{2}{*}{ Female } & KDC & \begin{tabular}{|l|}
1202 \\
\end{tabular} & 2.6 & 0.1 & 260 & 2.6 & \begin{tabular}{|l|}
0.1 \\
\end{tabular} & 261 & 2.6 & \begin{tabular}{|l|}
0.1 \\
\end{tabular} & 182 & 2.6 & 0.1 & 133 & 2.7 & 0.2 & $<0.001$ & 0.15 & 0.00 & $<0.001$ & 0.13 & \begin{tabular}{|l|}
0.00 \\
\end{tabular} & $<0.001$ \\
\hline & & & & \begin{tabular}{|l|} 
Ansan- \\
Ansung \\
\end{tabular} & 2775 & 2.6 & 0.1 & 228 & 2.5 & 0.1 & 1215 & 2.6 & 0.1 & 818 & 2.6 & 0.1 & 514 & 2.6 & 0.1 & $<0.001$ & 0.13 & 0.00 & $<0.001$ & 0.13 & 0.00 & $<0.001$ \\
\hline \multirow{4}{*}{ (n) } & & & & KDC & 682 & 2.6 & \begin{tabular}{|l|}
0.2 \\
\end{tabular} & 145 & 2.6 & 0.2 & 156 & 2.6 & 0.2 & 111 & 2.6 & 0.2 & 76 & 2.5 & 0.2 & $<0.01$ & -0.13 & 0.00 & $<0.01$ & -0.13 & \begin{tabular}{|l|}
0.00 \\
\end{tabular} & $<0.01$ \\
\hline & NTP & Nas & Male & \begin{tabular}{|l|} 
Ansan- \\
Ansung
\end{tabular} & 2578 & 2.6 & 0.2 & 248 & 2.6 & 0.2 & 1238 & 2.6 & 0.1 & 669 & 2.5 & 0.2 & 423 & 2.5 & 0.2 & $<0.001$ & -0.11 & 0.00 & $<0.001$ & -0.12 & 0.00 & $<0.001$ \\
\hline & $(\mathrm{H})$ & & & KDC & 1183 & 2.5 & 0.2 & 260 & 2.5 & \begin{tabular}{|l|}
0.1 \\
\end{tabular} & 254 & 2.5 & \begin{tabular}{|l|}
0.2 \\
\end{tabular} & 183 & 2.5 & 0.2 & 124 & 2.4 & 0.2 & $<0.001$ & -0.24 & 0.00 & $<0.001$ & -0.22 & \begin{tabular}{|l|}
0.00 \\
\end{tabular} & $<0.001$ \\
\hline & & & Female & \begin{tabular}{|l|} 
Ansan- \\
Ansung
\end{tabular} & 2814 & 2.4 & 0.2 & 227 & 2.4 & 0.2 & 1229 & 2.5 & 0.2 & 829 & 2.4 & 0.2 & 529 & 2.4 & 0.2 & $<0.001$ & -0.15 & 0.00 & $<0.001$ & -0.15 & 0.00 & $<0.001$ \\
\hline & & & & KDC & 697 & 136.5 & \begin{tabular}{|l|}
10.3 \\
\end{tabular} & 153 & 138.7 & 10 & 156 & 138 & 9.5 & 116 & 135.6 & 9.9 & 74 & 134.5 & 11.5 & $<0.001$ & -0.19 & 0.04 & $<0.001$ & -0.16 & \begin{tabular}{|l|}
0.04 \\
\end{tabular} & $<0.001$ \\
\hline 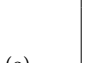 & 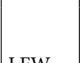 & Lower facial & Male & \begin{tabular}{|l|} 
Ansan- \\
Ansung
\end{tabular} & 2592 & 138.1 & 9.7 & 246 & 140.5 & 8.8 & 1245 & 140.1 & 9.2 & 674 & 136.1 & 9.7 & 427 & 134 & 9.9 & $<0.001$ & -0.26 & 0.02 & $<0.001$ & -0.13 & 0.02 & $<0.001$ \\
\hline (o) & LFW & & & KDC & 1256 & \begin{tabular}{|l|}
128.4 \\
\end{tabular} & 8.7 & 270 & \begin{tabular}{|l|}
128.9 \\
\end{tabular} & \begin{tabular}{|l|}
8.3 \\
\end{tabular} & 269 & 129 & \begin{tabular}{|l|}
8.3 \\
\end{tabular} & 193 & \begin{tabular}{|l|}
129.6 \\
\end{tabular} & 9.2 & 142 & \begin{tabular}{|l|}
128.7 \\
\end{tabular} & 9.7 & 0.805 & 0.00 & 0.03 & 0.96 & -0.07 & \begin{tabular}{|l|}
0.02 \\
\end{tabular} & $<0.05$ \\
\hline & & & Female & \begin{tabular}{l|} 
Ansan- \\
Ansung
\end{tabular} & 2971 & 130.5 & 8 & 246 & 131 & 7.7 & 1280 & 131.2 & 7.5 & 868 & 130.5 & 8.1 & 577 & 128.4 & 8.7 & $<0.001$ & -0.12 & 0.02 & $<0.001$ & -0.13 & 0.01 & $<0.001$ \\
\hline
\end{tabular}

Table 1. Means and standard deviations of facial metrics in KDC and Ansan-Ansung populations.

(A) Palpebral fissure height Male in Female

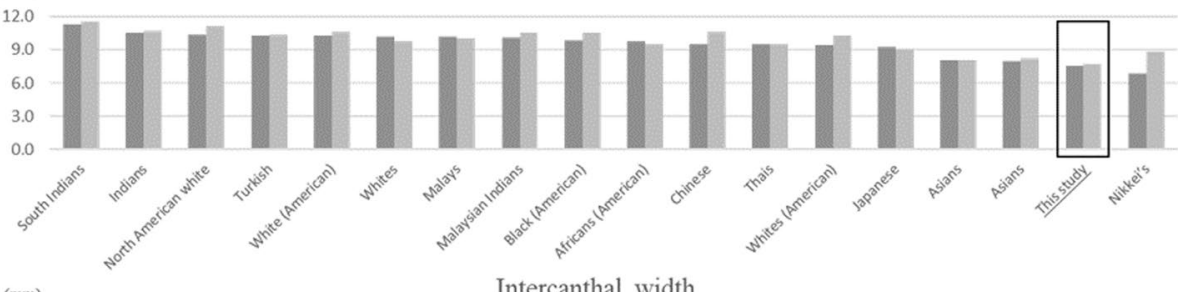

(B) $(\mathrm{mm} /$ Intercanthal width

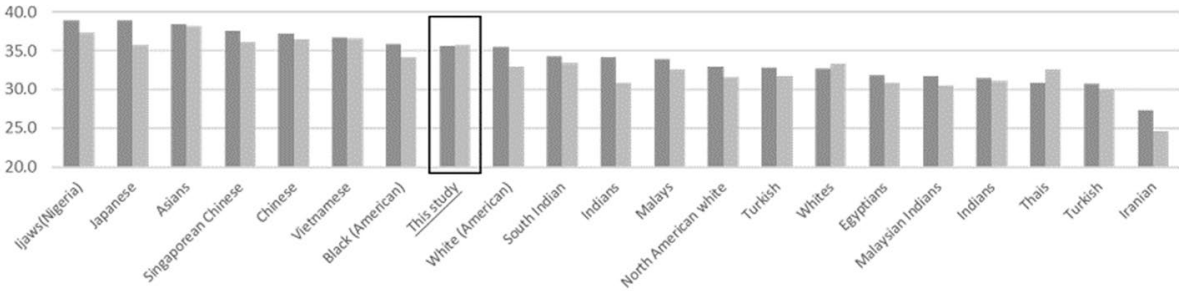

(C)

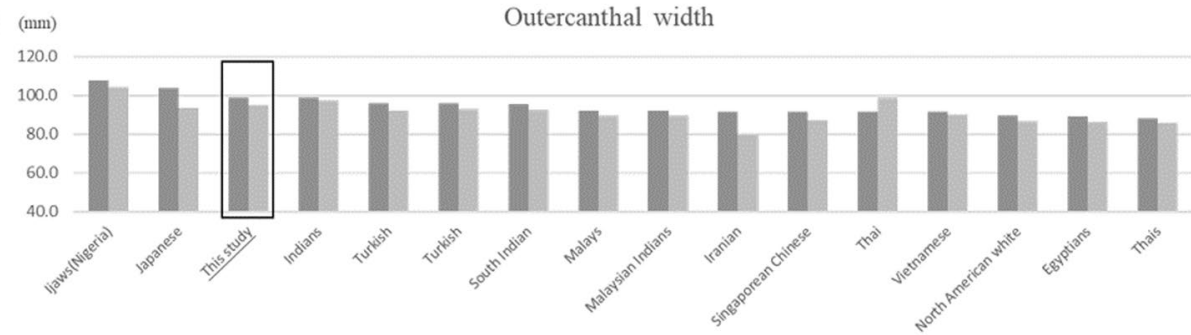

Figure 2. Ethnic comparison of the human orbital region. (A) Palpebral fissure height, (B) intercanthal width, and (C) outer canthal width. The data on ethnicities other than Korean were obtained from Vasanthakumar et al. ${ }^{14}$. 


\begin{tabular}{|c|c|c|c|c|c|c|c|c|c|c|}
\hline \multirow[b]{2}{*}{ Phenotypes } & \multirow[b]{2}{*}{ Population } & \multicolumn{5}{|l|}{ Total } & \multicolumn{2}{|c|}{ Male } & \multicolumn{2}{|c|}{ Female } \\
\hline & & $N$ & Mean & $S D$ & $N$ & Mean & $S D$ & $N$ & Mean & $S D$ \\
\hline \multirow{2}{*}{ BMI $\left(\mathrm{kg} / \mathrm{m}^{2}\right)$} & KDC & 1,408 & 23.8 & 3.1 & 724 & 24.0 & 3.1 & 1,284 & 23.1 & 3.3 \\
\hline & Ansan-Ansung & 5,643 & 24.4 & 3.1 & 2,648 & 24.3 & 2.9 & 2,995 & 24.6 & 3.2 \\
\hline \multirow{2}{*}{ Age (years) } & KDC & 1,408 & 57.1 & 10.8 & 724 & 56.9 & 10.5 & 1,284 & 57.2 & 11.0 \\
\hline & Ansan-Ansung & 5,643 & 60.4 & 8.5 & 2,648 & 59.9 & 8.4 & 2,995 & 60.9 & 8.6 \\
\hline
\end{tabular}

Table 2. Study Population Characteristics.

\section{Discussion}

In this study, we analysed 15 facial metrics for gender differences and for ageing-related changes in two large Korean cohorts. Among these 15 metrics, 12 showed gender differences and tendencies toward age-related change. Increasing tendencies were observed in $\mathrm{BRH}, \mathrm{ULH}, \mathrm{NTH}$, and PNL, and decreasing tendencies were observed in OW, RPFH, LPFH, RULT, LULT, NTP, FBW, and LFW. The decreasing tendencies were not changed even when adjusted for body mass index, indicating that some other structures under the skin (such as collagen and muscle) were reduced in the aged population.

In the orbital region, BRH showed a tendency to increase with age in both the KDC and Ansan-Ansung cohorts. The $\mathrm{BRH}$ is the nodule or crest of bone situated on the frontal bone of the skull ${ }^{10}$; this ridge separates the forehead itself from the tops of the eye sockets. Normally, in humans, the ridges arch over each eye, offering mechanical protection ${ }^{11}$. Typically, the arches are more prominent in men than in women and vary between different ethnic groups ${ }^{12}$.

"RPFH" and "LPFH" are abbreviations for the right and left palpebral fissure height. The palpebral fissure is the elliptic space between the medial and lateral canthi of the two open eyelids. There are many studies of palpebral fissure height; this metric is approximately $10 \mathrm{~mm}$ in adults and is smaller in East Asian populations than in white populations ${ }^{13,14}$. The gender differences in palpebral fissure height have been well summarized in reports on a South Indian population ${ }^{14}$. The bilateral orbital region, which is part of the upper face, acts as a key determinant of the perception of facial attractiveness, youthfulness and health ${ }^{14}$. Our comparison of palpebral fissure height implies that a very small PFH is a typical Korean facial characteristic (Fig. $2 \mathrm{~A}$ ). IW did not show any alteration with age, but OW showed a prominent decreasing pattern. The ethnic comparison of Fig. 2B,C showed that our study group had a shorter IW than Japanese or Chinese individuals, but the gender difference was no more prominent in Koreans than in other ethnic groups. In contrast, the OW of our study population was relatively large and was similar to that of the Japanese population. Therefore, the characteristics of the orbital region of Koreans are a small palpebral height and a wide canthal region. In the nasal area, the NTH slightly increased with age, and the NTP slightly decreased with age, but the NBH did not show any tendency to change. Additionally, the profile nasal length increased slightly with ageing only in males. The nasal tip is a challenging part of the nose for plastic surgeons because of variations in the anatomy of the lower lateral cartilage ${ }^{15}$. Therefore, our results help to clarify the direction of surgical correction for nasal tip deformities.

In the orthodontic region, the ULH slightly increased with ageing, and the RULT and LULT showed slight decreases with ageing. The upper lip is an important attractive point for the smile. According to Hulsey, the "smile is one of the most effective means by which people convey their emotions" ${ }^{16}$. The ULH is affected by the gingival margin of the upper central incisors and influences the attractiveness of a smile ${ }^{16}$. Generally, a shorter ULH indicates a higher smile line. On average, the smile line was found to be $1.5 \mathrm{~mm}$ higher in women than in $\mathrm{men}^{17}$; similarly, in the current study, the ULH was greater in males than in women, indicating a shorter smile line in women (see Table 1g). Although the concept of beauty has changed throughout the centuries, the thickness of the upper lip has always been a subject of interest and has importance in every culture ${ }^{18}$. These results may be beneficial to forensic anthropologists, plastic and reconstructive surgeons, and orthodontists.

Regarding overall facial width, both FBW and LFW slightly decreased with ageing, and the tendency was more prominent in male than female subjects. Facial width is reportedly associated with testosterone levels in males ${ }^{19}$. Therefore, the decrease in testosterone in elderly males may decrease the facial width.

\section{Conclusion}

Our study found that 12 of 15 facial metrics changed with age in subjects of both genders. The changes in eye size, nose length, upper lip thickness, and facial width might be general trends in Koreans because of the consistent tendencies in two large independent cohorts. Changes in facial metrics could serve as indicators to predict age from photographs and would be helpful for aesthetic facial care. To our knowledge, there are very few studies on the alteration of facial metrics with ageing, particularly in groups over 40 years old. Moreover, our study did not analyse the facial ageing of other ethnic populations. Therefore, we hope that the currents results will be validated in other ethnic groups and applied to diverse fields.

\section{Materials and Methods}

Study participants. A total of 1,926 participants in the KDC cohort were recruited from 19 sites (Korean Oriental Medical Clinics) between 2007 and $2010^{6}$, and a total of 5,643 participants in the Ansan-Ansung cohorts were recruited from two regions in southern Korea from 2009 to 2012 for the Korean Genome and Epidemiology Study (KoGES) $)^{6,20}$ (Table 2). The subjects were photographed with a neutral expression in both frontal and profile views under the following standard conditions: the hair should be pulled back with a hair band; the centre points of the two pupils should be horizontally aligned, as should the upper auricular perimeters; and a ruler should be 
placed approximately $10 \mathrm{~mm}$ below the chin to convert pixels into millimetres. All participants provided written informed consent to participate in the study. This protocol was approved by the Korea Institute of Oriental Medicine Institutional Review Board (I-0910/02-001), and all research was performed in accordance with the relevant guidelines/regulations.

Craniofacial measurements. Detailed descriptions of candidate feature variables and the corresponding measurement methods have been provided in a previous report ${ }^{6,9}$. Briefly, the facial variables were limited to those that could be easily quantified. Facial feature points in frontal and lateral images were automatically extracted by detecting and analysing the face, eyes, nose, mouth, and contours via an in-house program in Visual Studio C ++ using OpenCV (Open Source Computer Vision Library). The positions of the extracted points were confirmed by a well-trained operator (accuracy: $98.8 \%$ on average). Fifteen facial metrics, each defined by the distance between two facial points, were derived from the photographs by converting pixels into millimetres using MATLAB software ${ }^{21}$.

For statistical analysis, 5 severely skewed facial variables, including PNL, NTH(V), NTP(H), RULT, and LULT, were ln transformed. We removed the outliers, which were defined using the first and third quartiles and the interquartile range of each facial variable. In each facial variable, measurements below the first quartile $-2.0 \times$ interquartile range or above the third quartile $+2.0 \times$ interquartile range were defined as outliers and excluded.

Statistical analysis. For each facial variable, the mean length was compared between age groups ( $40 \mathrm{~s}$ vs $50 \mathrm{~s}, 60 \mathrm{~s}$ or $70+$ ) using Student's t-test. Additionally, the mean lengths of male and female facial variables were plotted (Fig. 1) for both the KDC and Ansan-Ansung cohorts. We used the following criteria to identify phenotypes that tended to change with age: the mean underwent a significant gradual increase or decrease $(\mathrm{p}<0.05)$, and the pattern appeared to be similar between the KDC and Ansan-Ansung cohorts.

\section{References}

1. Enlow, D. H. Facial Growth. 3rd edn, (SPCK Publishing, 1990).

2. Kolar, J. C. \& Salter, E. M. Craniofacial Anthropometry: Practical Measurement of the Head and Face for Clinical, Surgical, and Research Use. 1st edn, (Charles C Thomas Pub Ltd, 1997).

3. Huxley, J. S. \& TEISSIER, G. Terminology of Relative Growth. Nature 137, 780-781, https://doi.org/10.1038/137780b0 (1936).

4. Chai, Y. \& Maxson, R. E. Jr. Recent advances in craniofacial morphogenesis. Developmental dynamics: an official publication of the American Association of Anatomists 235, 2353-2375, https://doi.org/10.1002/dvdy.20833 (2006).

5. Machado, C. E. P. et al. A new approach for the analysis of facial growth and age estimation: Iris ratio. PloS one 12, e0180330, https:// doi.org/10.1371/journal.pone.0180330 (2017).

6. Cha, S. et al. Identification of five novel genetic loci related to facial morphology by genome-wide association studies. BMC genomics 19, 481, https://doi.org/10.1186/s12864-018-4865-9 (2018).

7. Lundstrom, A. \& McWilliam, J. Comparison of some cephalometric distances and corresponding facial proportions with regard to heritability. European journal of orthodontics 10, 27-29 (1988).

8. Cattaneo, C. et al. Can facial proportions taken from images be of use for ageing in cases of suspected child pornography? A pilot study. International journal of legal medicine 126, 139-144, https://doi.org/10.1007/s00414-011-0564-7 (2012).

9. Do, J. H., Ku, B., Jang, J. S., Kim, H. \& Kim, J. Y. Analysis of Sasang constitutional types using facial features with compensation for photographic distance. Integrative medicine research 1, 26-35, https://doi.org/10.1016/j.imr.2012.09.003 (2012).

10. Oyen, O. J., Rice, R. W. \& Cannon, M. S. Browridge structure and function in extant primates and Neanderthals. American Journal of Physical Anthropology 51, 83-95, https://doi.org/10.1002/ajpa.1330510111 (1979).

11. Endo, B. Distribution of stress and strain produced in the human face by masticatory forces. Journal of Anthropological Society of Nippon 73, 123-136, https://doi.org/10.1537/ase1911.73.123 (1965).

12. Shearer, B. M., Sholts, S. B., Garvin, H. M. \& Warmlander, S. K. Sexual dimorphism in human browridge volume measured from 3D models of dry crania: a new digital morphometrics approach. Forensic science international 222(400), e401-405, https://doi. org/10.1016/j.forsciint.2012.06.013 (2012).

13. Packiriswamy, V., Kumar, P. \& Bashour, M. Anthropometric and Anthroposcopic Analysis of Periorbital Features in Malaysian Population: An Inter-racial Study. Facial plastic surgery: FPS 34, 400-406, https://doi.org/10.1055/s-0038-1648224 (2018).

14. Vasanthakumar, P., Kumar, P. \& Rao, M. Anthropometric analysis of palpebral fissure dimensions and its position in South Indian ethnic adults. Oman medical journal 28, 26-32, https://doi.org/10.5001/omj.2013.06 (2013).

15. Farahvash, M. R., Ebrahimi, A., Farahvash, B. \& Farahvash, Y. Anatomic and anthropometric analysis of 72 lower lateral nasal cartilages from fresh Persian (Iranian) cadavers. Aesthetic surgery journal 32, 447-453, https://doi.org/10.1177/1090820X12442084 (2012).

16. Hulsey, C. M. An esthetic evaluation of lip-teeth relationships present in the smile. American journal of orthodontics 57, 132-144 (1970).

17. Rigsbee, O. H. 3rd, Sperry, T. P. \& BeGole, E. A. The influence of facial animation on smile characteristics. The International journal of adult orthodontics and orthognathic surgery 3, 233-239 (1988).

18. Bozdag, Z. K., Kurkcuoglu, A., Ustdal, A., Cam, Y. \& Oguz, O. Upper and Lower Lip Soft Tissue Thicknesses Differ in Relation to Age and Sex. Int J Morphol 35, 852-858 (2017).

19. Lefevre, C. E., Lewis, G. J., Perrett, D. I. \& Penke, L. Telling facial metrics: facial width is associated with testosterone levels in men. Evol Hum Behav 34, 273-279, https://doi.org/10.1016/j.evolhumbehav.2013.03.005 (2013).

20. Kim, Y., Han, B. G. \& Ko, G. E. S. G. Cohort Profile: The Korean Genome and Epidemiology Study (KoGES) Consortium. International journal of epidemiology 46, e20, https://doi.org/10.1093/ije/dyv316 (2017).

21. Lee, B. J., Do, J. H. \& Kim, J. Y. A classification method of normal and overweight females based on facial features for automated medical applications. J Biomed Biotechnol 2012, 834578 (2012).

\section{Acknowledgements}

We are grateful to the Korean Oriental Medicine Institute for providing the facial metric analysis results. This study was supported by the National Research Foundation in Korea (NRF-2018 M3E3A1057354 and 2018 M3E3A1057298). 


\section{Author Contributions}

Hae-Young Lee: manuscript writing. Seongwon Cha \& Hyo-Jeong Ban: data collection, statistics. In-Young Kim \& Bo-Reum Park: data management, figure drawing. Ig-Jae Kim: data collection, statistics. Kyung-Won Hong: data analysis, manuscript writing.

\section{Additional Information}

Competing Interests: The authors declare no competing interests.

Publisher's note Springer Nature remains neutral with regard to jurisdictional claims in published maps and institutional affiliations.

(c) (i) Open Access This article is licensed under a Creative Commons Attribution 4.0 International License, which permits use, sharing, adaptation, distribution and reproduction in any medium or format, as long as you give appropriate credit to the original author(s) and the source, provide a link to the Creative Commons license, and indicate if changes were made. The images or other third party material in this article are included in the article's Creative Commons license, unless indicated otherwise in a credit line to the material. If material is not included in the article's Creative Commons license and your intended use is not permitted by statutory regulation or exceeds the permitted use, you will need to obtain permission directly from the copyright holder. To view a copy of this license, visit http://creativecommons.org/licenses/by/4.0/.

(C) The Author(s) 2019 